\title{
Formación del profesorado de Educación Física en TIC: Modelo TPACK
}

Training physical education teachers in ICT: TPACK model

\author{
CARLOS FERNÁNDEZ-ESPÍNOLA \\ Departamento de Didácticas Integradas. Universidad de Huelva \\ LAURA LADRÓN-DE-GUEVARA \\ Centro de Estudios Universitario Cardenal Spínola CEU \\ BARTOLOMÉ J. ALMAGRO \\ Departamento de Didácticas Integradas. Universidad de Huelva \\ JOSÉ ANTONIO REBOLLO \\ Departamento de Didácticas Integradas. Universidad de Huelva
}

Recibido: $28 / 05 / 2018$

Aceptado: 25/06/2018

PALABRAS CLAVES

Tecnologías de la Información y la Comunicación, Educación Física, Educación Primaria, Docentes, TPACK, Autoeficacia computacional.

\section{ABSTRACT}

The main aim of this study was to analyze the training in ICT of Primary Physical Education teachers, according to this model. The Spanish translation of the TPACK survey and Computer Self-Efficacy scale was administered along items to measure the Internet use. A sample of 60 physical education teachers from 40 schools in Seville and Huelva were selected. The results revealed that TPACK perceptions of physical education teachers were at satisfactory levels, especially in the pedagogical component and content knowledge. Also, the teachers showed acceptable levels in the computer self-efficacy scale, by means of using the Internet daily with a social purpose rather than professional. Positive correlations were found in terms of the most important variables of the study (4 types of knowledge of the TPACK and self-efficacy in the use of ICT). Specifically, the technological knowledge (TK, PCK+TPK and TPACK) correlations with computer self-efficacy were higher.

Para citar este artículo: Fernández Espínola, C., Ladrón-de-Guevara, L. Almagro, B. J. y Rebollo-González, J. A. . (2018). Fomación del profesorado de Educación Física en TIC: Modelo TPACK. EA, Escuela Abierta, 21, 65-75. doi:10.29257/EA21.2018.05

\section{KEYWORDS}

Information and Communications Technologies, Physical Education, Elementary Education, Teachers, TPACK, Computer Self-efficacy. 


\section{INTRODUCCIÓN}

En la sociedad de la información y la comunicación no se puede dudar del papel fundamental que tienen las tecnologías digitales en el ámbito educativo (Sevillano y Fuero, 2013). Sin embargo, durante un gran periodo de tiempo, las políticas educativas que se encargan de incorporar las tecnologías de la información y la comunicación (de ahora en adelante TIC) en los centros educativos han estado más preocupadas de incrementar la dotación de recursos y medir los usos de la tecnología que de tomar medidas para mejorar la formación permanente del profesorado. En este sentido, una de las principales medidas que puede asegurar el uso de las TIC en los centros, es que progrese la formación de los docentes en relación a la tecnología (Fernández-Díaz y Calvo, 2012).

\subsection{Formación TIC del profesorado}

En los últimos años, los centros educativos han presentado dificultades para integrar y aprovechar los recursos TIC de manera adecuada tanto en el aula, como en la formación del profesorado y en la evaluación de la repercusión que tiene el uso de las tecnologías en el proceso de enseñanza-aprendizaje (Pérez-Rodríguez, Aguaded y Fandos, 2010).

El creciente uso de la tecnología multimedia y la entrada de las web 2.0 han cambiado los contextos y las formas de aprender, incrementando también las oportunidades para el aprendizaje (Rebollo-Catalán, Vico-Bosch y García-Pérez, 2015).

Las redes sociales son otro recurso que permite el aprendizaje colaborativo dentro de una educación formal. Sin embargo, es más habitual que para aprender a usar redes sociales y aprender más sobre TIC se acuda con mayor frecuencia a contextos informales que formales (Rebollo-Catalán et al., 2015).

En este sentido, la escritura científica ha informado que no solo el contexto de aprendizaje es un factor importante para usar las redes sociales sino también los motivos de ese uso. Según Jiménez-Cortés, Rebollo-Catalán, García-Pérez y Buzón-García (2015), los motivos principales por los que se usan las redes sociales son: relacionarse con los demás, mantenerse informados y entretenerse.

\subsection{Autoeficacia percibida en el uso de las TIC}

La autoeficacia percibida hace referencia a "los juicios de cada individuo sobre sus propias capacidades, en base a los cuales organizará y ejecutará sus actos de modo que le permitan alcanzar el rendimiento deseado" (Bandura, 1987, p. 416). En otras palabras, es un juicio personal sobre las capacidades propias para alcanzar una meta de la forma más exitosa posible. La autoeficacia también es un elemento de motivación que repercute en los sujetos aumentando el esfuerzo y la perseverancia para no desistir frente a dificultades (López, Sanabria y Sanabria, 2014).

Existe un término que relaciona las TIC con la autoeficacia percibida, se trata de la autoeficacia computacional. Este concepto se define como "el juicio subjetivo sobre las habilidades computacionales que posee la persona"(Durán-García y Durán-Aponte, 2013, p. 249), es decir, las habilidades que una persona posee con respecto a un ordenador y la informática. 
Semiz e Ince (2012) y Cengiz (2014) en sus estudios, proponen identificar y examinar las relaciones entre la autoeficacia tecnológica, las expectativas de resultados y el conocimiento tecnológico pedagógico del contenido con respecto a las TIC en los docentes de Educación Física (EF).

\subsection{Conocimiento Tecnológico, Pedagógico y de Contenido: El modelo TPACK}

El uso de las TIC por parte de los docentes debe estar dirigido hacia tres componentes del conocimiento: tecnológico, pedagógico y disciplinar o conceptual (Cózar, Zagalaz y Sáez, 2015). El modelo Technological Pedagogical Content Knowledge (TPACK), encaja en este planteamiento.

Este modelo tiene su origen con Shulman (1987), que propone la existencia de un cierto dominio del conocimiento didáctico que se va a impartir, en el cual se incluye tanto la pedagogía (métodos de enseñanza, necesidades de los estudiantes, preparación, etc.) como el contexto en el que enseñan los docentes (Semiz y Ince, 2012). Sirviéndose de esta idea de Shulman, surge el modelo TPACK, elaborado y popularizado años después por Mishra y Koehler (2006) y Mishra y Koehler (2008).

De acuerdo con este modelo, para que un profesor esté capacitado para incorporar las TIC en las aulas, no es suficiente con tener dominio en estas tres áreas de conocimiento por separado, sino que debe ser capaz de comprenderlas y percibirlas como elementos que se interrelacionan e interaccionan entre sí (Cabero, 2014; Cabero y Barroso, 2016). La comprensión de este conocimiento está por encima y más allá de entender la tecnología, el contenido, o la pedagogía aisladamente, sino más bien como una forma emergente que entiende cómo estas formas de conocimiento interactúan unos con otros (Mishra y Koehler, 2008).

En España, algunos autores (Cabero, 2014; Cabero, Marín y Castaño, 2015; Roig y Flores, 2014) han tratado de analizar la formación del profesorado en TIC; para ello seleccionaron uno de los cuestionarios más usado para dictaminar el modelo TPACK del profesorado, en concreto el elaborado por Schmidt et al. (2009). Dicho cuestionario estaba diseñado para cuatro áreas temáticas diferentes: matemáticas, lenguaje, ciencias y ciencias sociales.

Semiz e Ince (2012) adaptaron el cuestionario al área de EF con la ayuda de un experto en actividad física con un doctorado en el campo de la pedagogía deportiva. Desde entonces, a nivel internacional algunos estudios han empezado a prestar atención a la formación del profesorado en EF con respecto a las TIC, empleando el modelo TPACK para evaluar el conocimiento tecnológico, pedagógico y del contenido de los docentes (Arslan, 2015; Cengiz, 2014; Juniu, 2011). En España, son pocos los estudios que se han centrado en analizar el uso de las TIC por parte del profesorado de EF (Prat, Camerino, y Coiduras, 2013) o la formación en competencias tecnológicas de los docentes de EF (Blasco, Mengual y Roig, 2007; Pla-Campàs y Juncà, 2015).

El objetivo principal de este estudio fue analizar la formación TIC del profesorado de EF de Educación Primaria, de acuerdo con el modelo TPACK, y el nivel de autoeficacia percibida que poseen los docentes de EF en activo, estudiando las relaciones entre estas variables. Otro propósito fue describir la frecuencia, medios y motivos por los cuales los docentes de EF utilizan Internet y las redes sociales. 


\section{MÉTODO}

\subsection{Diseño}

El presente estudio corresponde a un diseño selectivo transversal, por consiguiente, se trata de una investigación empírica con estrategia descriptiva, respondiendo a un muestreo intencional o de conveniencia (Ato, López y Benavente, 2013).

\subsection{Muestra del estudio}

La muestra estuvo formada por 60 docentes de EF en la etapa de Educación Primaria pertenecientes a 40 centros educativos de las provincias de Sevilla y Huelva. De los cuales eran 42 hombres y 18 mujeres, de edades comprendidas entre los 27 y los 58 años $(M=41$; DT = 7,67), y con una media de antigüedad impartiendo clases de 14,5 años (DT = 7,99). En concreto, 37 docentes eran de centros de Sevilla y 23 de Huelva. La mayoría de los encuestados tenían de formación inicial la diplomatura en EF (73,33 \%) y el 51,67\% poseía formación específica en TIC (cursos en pizarras digitales, Guadalinex, diseño de páginas web, blogs, etc.).

\subsection{Instrumentos}

Para la recogida de datos se utilizaron tres cuestionarios encabezados por una primera parte con cuestiones sobre información sociodemográfica en la que se preguntó el género, la edad, el centro en el que imparten clases, años de antigüedad como docentes y formación del profesorado.

\section{Preguntas sobre el uso de Internet.}

Con el objetivo de describir los motivos, dispositivos y frecuencia con que utilizan las TIC, se realizaron cuestiones de elaboración propia, en las que se preguntó la frecuencia con la que usan Internet a la semana y a diario, dispositivo que utilizan para conectarse, redes sociales que usan habitualmente y motivo de su uso.

\section{Cuestionario TPACK para docentes de Educación Física.}

Los diferentes tipos de conocimientos que tienen los docentes se abordaron con una versión en castellano del cuestionario TPACK para docentes de EF (Ladrón-de-Guevara, Almagro y Cabero, en revision). Dicho cuestionario está formado por 30 ítems distribuidos en 4 tipos de conocimientos, con una escala de respuesta tipo Likert de 1 a 5 , donde 1 correspondía a muy en desacuerdo y 5 a muy de acuerdo. La distribución de los ítems según el tipo de conocimiento fue como se detalla a continuación: conocimiento tecnológico (TK; 7 ítems); conocimiento del contenido (CK; 3 ítems); conocimiento pedagógico y conocimiento pedagógico del contenido (PK + PCK; 8 ítems); conocimiento tecnológico del contenido, conocimiento tecnológico pedagógico y conocimiento tecnológico pedagógico del contenido (TPK + TCK + TPACK; 12 ítems). En este estudio se obtuvieron valores del alfa de Cronbach de 0,92 para el factor TK; 0,94 para el CK; 0,94 para el PK + PCK; y de 0,92 para TPK + TCK + TPACK. 


\section{Escala de autoeficacia percibida en el uso del ordenador.}

Por último, para identificar el nivel de autoeficacia percibida en el uso de las TIC por parte de los docentes de EF se usó una versión traducida al castellano (Rebollo-Catalán et al., 2015) de la Computer Self-Efficacy Scale (Howard, 2014). La escala está formada por 12 ítems con una escala de respuesta tipo Likert, con cinco opciones de respuesta que oscilaba entre 1 (muy en desacuerdo) y 5 (muy de acuerdo). La consistencia interna medida a través del alfa de Cronbach fue de 0,96 para este estudio.

\subsection{Procedimiento}

En primer lugar, se prepararon los cuestionarios para poder administrarlos de forma presencial y de forma telemática. Para comenzar la recogida de datos se contactó con docentes de EF, explicándoles el objetivo del estudio y solicitándoles su colaboración. La recogida de datos fue distinta para las dos provincias en las que se obtuvo la muestra de la investigación. En Sevilla durante la distribución de los cuestionarios estuvo presente el investigador principal o terceras personas capacitadas para solventar todas las dudas a la hora de rellenar el cuestionario. En Huelva, por el contrario, se envió el mismo cuestionario formato on-line.

\subsection{Análisis de los datos}

En primer lugar, se depuró la matriz de datos y se calculó la fiabilidad de los instrumentos. Posteriormente, se realizó un análisis descriptivo y de correlaciones bivariadas entre las principales variables del estudio. Los diferentes análisis se llevaron a cabo con el programa estadístico SPSS 23.

Tabla 1

\begin{tabular}{|c|c|c|}
\hline VARIABLES & $\begin{array}{c}\text { CATEgorías/VAlores EXTRE- } \\
\text { MOS }\end{array}$ & Media/Porcentajes \\
\hline \multirow[t]{2}{*}{ Frecuencia de uso de Internet (Semanal) } & $\operatorname{Min}=1$ & $6,66(\mathrm{DT}=1,03)$ \\
\hline & $\operatorname{Max}=7$ & \\
\hline \multirow[t]{2}{*}{ Frecuencia de uso de Internet (Diario) } & $\operatorname{Min}=10$ & $105,5(\mathrm{DT}=87,09)$ \\
\hline & $\operatorname{Max}=500$ & \\
\hline \multirow[t]{7}{*}{ Dispositivo de uso } & Solo móvil & $1,67 \%$ \\
\hline & Solo ordenador & $5 \%$ \\
\hline & Solo tablet & $0 \%$ \\
\hline & Móvil y ordenador & $35 \%$ \\
\hline & Móvil y tablet & $6,67 \%$ \\
\hline & Ordenador y tablet & $1,67 \%$ \\
\hline & Móvil, ordenador y tablet & $50 \%$ \\
\hline
\end{tabular}

Nota. DT = Desviación típica; Min = Valor mínimo; Max = Valor máximo. 


\section{RESULTADOS}

Como se puede ver en la Tabla 1 , los docentes encuestados utilizan Internet casi todos los días ( $\mathrm{M}=6,66$, DT $=1,03)$ y con un uso medio de 105,5 minutos al día $(\mathrm{DT}=87,09)$. También se puede observar que el $50 \%$ usa habitualmente tres dispositivos para conectarse a Internet (móvil, tablet y ordenador).

\section{Figura 1}

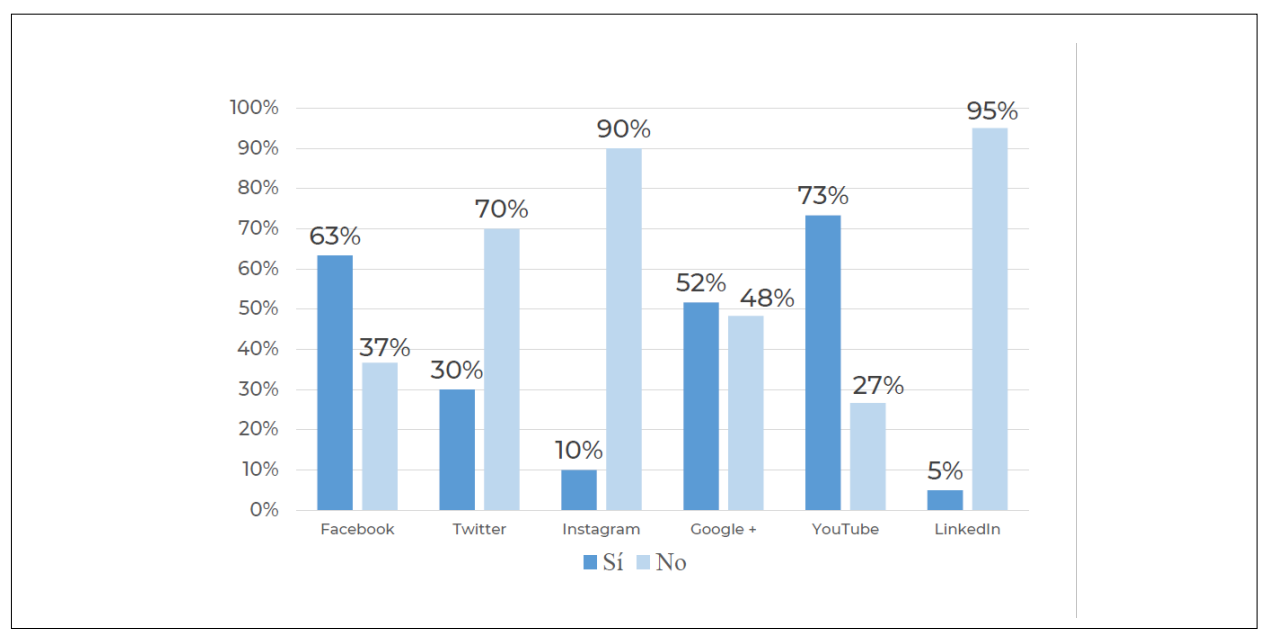

Los resultados muestran que las redes sociales que más usan los docentes de EF de esta muestra son YouTube, Facebook y Google + (ver Figura 1). Mientras que las redes sociales que menos emplean en orden decreciente son: Twitter (30\%), Instagram (10\%) y LinkedIn (5\%).

Los docentes de EF utilizan las redes sociales por varios motivos (ver Figura 2): el 65\% utiliza las redes para relacionarse con otras personas, el $42 \%$ les da uso con un perfil profesional, el $27 \%$ de los encuestados utiliza las redes sociales para promocionar su centro y un $18 \%$ hace uso de las redes sociales para expresarse abiertamente. Sólo el 22\% de los docentes utiliza las redes sociales con un motivo diferente (en la Figura 2, "Otros"), siendo las redes sociales fuente de información para su trabajo, como el más destacado.

En la Tabla 2 se muestran los estadísticos descriptivos (las puntuaciones medias y desviaciones típicas) y las correlaciones bivariadas obtenidas de los cuatro tipos de conocimientos que mide la versión para EF del TPACK, así como de la autoeficacia percibida en el uso del ordenador por parte de los docentes. La variable que obtuvo una puntuación media mayor fue el conocimiento del contenido $(M=4,27)$ seguida del conocimiento pedagógico y pedagógico del contenido $(\mathrm{M}=4,21)$. Como se puede observar en la Tabla 2, todas las variables correlacionaron de forma positiva y estadísticamente significativa entre ellas. En concreto, entre los conocimientos tecnológicos (TK, TCK + TPK + TPACK) y la autoeficacia computacional se encontraron las correlaciones más altas. 
Figura 2

Motivos para usar las redes sociales.

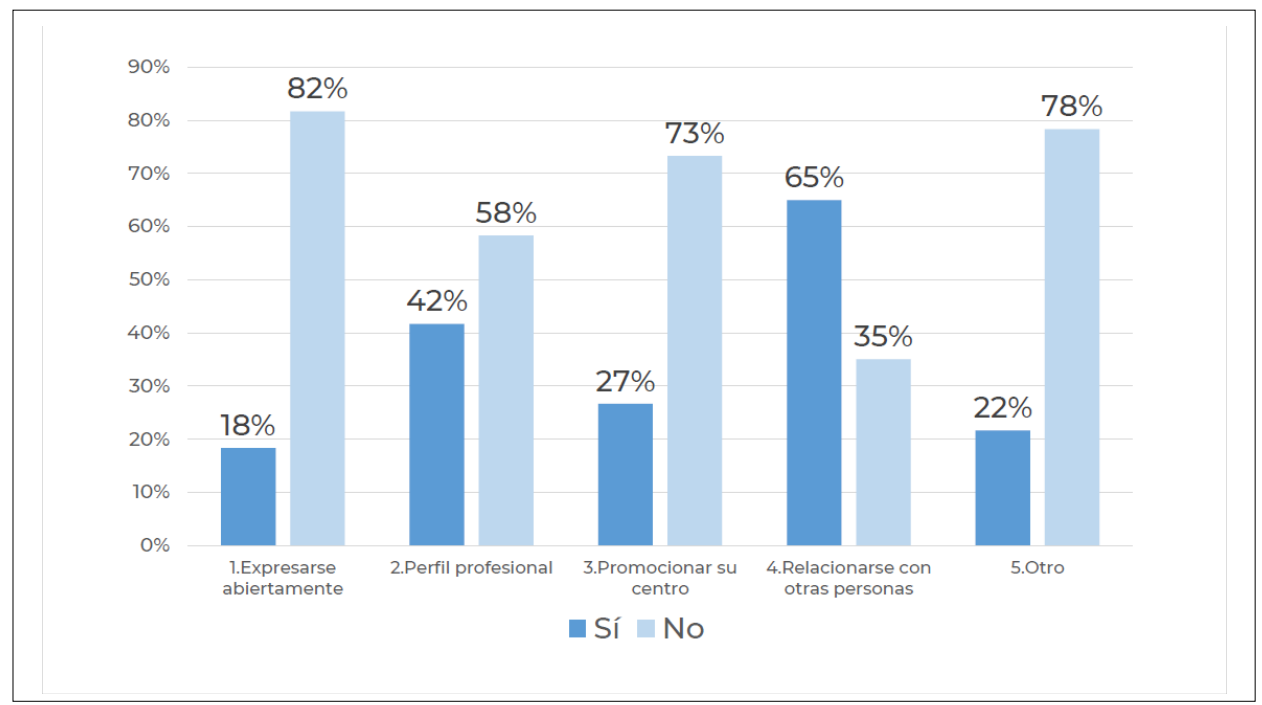

Tabla 2

Estadísticos descriptivos y correlaciones bivariadas

\begin{tabular}{|c|c|c|c|c|c|c|c|}
\hline VARIABLES & M & DT & 1 & 2 & 3 & 4 & 5 \\
\hline 1. Conocimiento Tecnológico (TK) & 3,51 & 0,77 & - & $0,49^{* *}$ & $0,49^{* *}$ & $0,79^{* *}$ & $0,81^{* *}$ \\
\hline 2. Conocimiento del Contenido $(\mathrm{CK})$ & 4,27 & 0,72 & - & - & $0,61^{* *}$ & $0,51^{* *}$ & $0,29 *$ \\
\hline $\begin{array}{l}\text { 3. Conocimiento Pedagógico y Conoci- } \\
\text { miento Pedagógico del Contenido }(\mathrm{PK}+ \\
\mathrm{PCK})\end{array}$ & 4,21 & 0,56 & - & - & - & $0,67^{* *}$ & $0,35^{* *}$ \\
\hline $\begin{array}{l}\text { 4. Conocimiento Tecnológico del Con- } \\
\text { tenido, Conocimiento Tecnológico Pe- } \\
\text { dagógico y Conocimiento Tecnológico } \\
\text { Pedagógico del Contenido (TCK + TPK + } \\
\text { TPACK) }\end{array}$ & 3,68 & 0,60 & - & - & - & - & $0,68^{* *}$ \\
\hline 5. Autoeficacia en el uso del ordenador & 3,42 & 0,80 & - & - & - & - & - \\
\hline
\end{tabular}




\section{DISCUSIÓN}

El objetivo principal del estudio fue analizar la formación TIC que tenían los docentes de EF de acuerdo al modelo TPACK y la autoeficacia percibida en el uso del ordenador. Los resultados muestran que los docentes se sienten competentes en los 4 tipos de conocimientos que se midieron con la versión española del cuestionario TPACK para EF (Ladrón-de-Guevara et al., en revisión), teniendo en cuenta que las puntuaciones superan en todos los casos una puntuación media de 3,5 puntos. Las puntuaciones medias más altas fueron en el conocimiento de contenido $(4,27)$, seguida muy de cerca por el conocimiento pedagógico y conocimiento pedagógico del contenido $(4,21)$, mostrando que los docentes perciben que su nivel de formación es alto en cuanto a recursos didácticos y conocimientos sobre el área de EF. Estos resultados van en la línea de lo encontrado por estudios realizados en otros países (e.g., Baert y Stewart, 2014; Cengiz, 2014; Semiz e Ince, 2012).

Se puede observar que la puntuación media más baja fue en el conocimiento tecnológico $(3,51)$. Asimismo, el factor formado por el Conocimiento Tecnológico del Contenido, Conocimiento Tecnológico Pedagógico y Conocimiento Tecnológico Pedagógico del Contenido (TCK + TPK + TPACK) no obtuvo una media muy alta $(3,68)$. Resultados que coinciden en parte con los obtenidos por Cabero (2014), en los cuales se obtuvo que los docentes que participaron en el estudio se autovaloraban de forma positiva en los 7 tipos de conocimientos que conforman el modelo TPACK, destacando sobre todo la puntuación más alta en conocimientos pedagógicos al igual que en este estudio. Por el contrario, los resultados obtenidos en el componente tecnológico y todas sus posibles combinaciones difieren con los de esta investigación, y es que el conocimiento tecnológico y sus uniones (TK, TCK, TPK, TPACK) mostraron puntuaciones más elevadas que el conocimiento del contenido y el conocimiento pedagógico del contenido.

En este sentido, Semiz e Ince (2012) destacan que aunque los niveles de conocimiento tecnológico pedagógico del contenido son satisfactorios, los docentes de EF no son buenos modelos en la utilización de la tecnología. Los resultados sugieren iniciar el camino hacia el cambio, ya que existe la necesidad de modificar la formación TIC centrada preferentemente en componentes tecnológicos (Cabero, 2014).

Por otro lado, Bustos (2012) defiende que el juicio de la persona sobre su capacidad para manejar ordenadores o recursos tecnológicos está íntimamente relacionado con la confianza de un docente para enseñar utilizando tecnología digital. En este sentido, en lo que respecta al nivel de autoeficacia percibida en el uso de los ordenadores por parte de los docentes de EF en activo, los resultados obtenidos sugieren que los docentes se sienten autoeficaces en diferentes acciones (e.g. en el manejo de programas informáticos), pero no con total seguridad (se obtuvo una media de 3,42 sobre 5 ).

Otro propósito del estudio era describir la frecuencia, medios y motivos por los cuales los docentes de EF utilizan Internet y las redes sociales. Los resultados muestran que los docentes de EF utilizan Internet prácticamente a diario $(M=6,66)$, coincidiendo con lo encontrado por Prat et al. (2013). En cuanto a los dispositivos que utilizan para conectarse a Internet, destaca que la mitad de los docentes encuestados utilizan tres dispositivos diferentes para navegar en la red: móvil, tablet y ordenador.

Por último, con respecto a las redes sociales, las más utilizadas por los docentes de EF son Facebook, YouTube y Google+. El motivo más destacado, por el que los docentes utilizan las redes sociales es principalmente para relacionarse con otras personas, coincidiendo con otros estudios en los que se mostraba que los motivos principales por los cuales se usan las redes sociales son prioritariamente relacionales (Rebollo-Catalán y Vico-Bosch, 2014; Jiménez-Cortés et al., 2015). Asimismo, Rebollo-Catalán et al. (2015) señalan que existen 
diferentes motivaciones para utilizar las redes sociales, destacando entre ellos motivos sociales, como reducir la distancia geográfica y relacionarse con diferentes personas.

En cuanto a los resultados del análisis de correlación, se encontraron correlaciones positivas y estadísticamente significativas entre los conocimientos tecnológicos (TK, PCK + TPK + TPACK) y la autoeficacia percibida en el uso de los ordenadores. En este sentido, otros estudios han mostrado anteriormente esta relación positiva entre la autoeficacia computacional percibida y el modelo TPACK (Cengiz, 2014; Semiz y Ince, 2012).

\section{CONCLUSIONES}

Tras analizar los resultados del estudio, podemos dar respuesta a los objetivos planteados, llegando a las siguientes conclusiones:

Los docentes de EF se sentían competentes en todos los niveles de conocimiento del modelo TPACK, sobresaliendo los conocimientos sobre el área de EF y sus orientaciones metodológicas para impartir una clase. Por otro lado, hay que destacar que en el conocimiento tecnológico y sus diferentes combinaciones se autovaloraban con menor puntuación que el resto. Esto nos lleva a la conclusión de que existe la necesidad de cambio, no hay que formar a los docentes en TIC de forma aislada, sino teniendo en cuenta el componente pedagógico y de contenido.

En cuanto a la autoeficacia percibida en relación a los ordenadores, los docentes de EF mostraron que son capaces de realizar múltiples acciones con un ordenador, pero sin llegar a percibirse como personas eficientes en el manejo del mismo. Por ello, hay que generar confianza en los docentes para que puedan realizar prácticas novedosas de poca transcendencia que conduzcan gradualmente a un cambio, posibilitando la integración de innovaciones de mayor envergadura.

Por los datos recogidos se pudo observar que los docentes de EF están muy vinculados a la tecnología en su vida diaria, utilizando Internet prácticamente a diario, conectándose a la red con hasta tres dispositivos diferentes y usando principalmente las redes sociales para relacionarse con otras personas. Podemos afirmar que la tecnología ha revolucionado el mundo social, sin embargo, aún necesita incorporarse con éxito al mundo de la enseñanza.

Finalmente, con respecto a futuras líneas de investigación, sería interesante realizar el estudio con docentes de EF de otros niveles educativos (secundaria y universitario), o realizar diseños cuasi-experimentales donde se impartiera un curso de formación en el uso de las TIC adaptado específicamente al área de EF y comprobar la efectividad de la intervención.

\section{REFERENCIAS}

Ato, M., López, J. J. y Benavente, A. (2013). Un sistema de clasificación de los diseños de investigación en psicología. Anales de Psicología, 29(3), 1038-1059. 
Arslan, Y. (2015). Determination of technopedagogical content knowledge competencies of preservice physical education teachers: A Turkish sample. Journal of Teaching in Physical Education, 34(2), 225-241. https://doi. org/10.1123/jtpe.2013-0054

Baert, H. y Stewart, A. (2014). The effects of role modeling on technology integration within physical education teacher education. JTRM in Kinesiology, 26, 1-26. Recuperado de https://eric.ed.gov/?id=EJ1053415

Bandura, A. (1987). Pensamiento y acción: Fundamentos sociales. Barcelona: Martínez Roca.

Blasco, J. E., Mengual, S. y Roig, R. I. (2007). Competencias tecnológicas en el espacio europeo de educación superior. Propuesta de formación del maestro especialista en educación física. Profesorado. Revista de Currículum y Formación del Profesorado, 11(2), 1-16. Recuperado de http://www.ugr.es/ recfpro/rev112ART10.pdf

Bustos, C. E. (2012). Creencias docentes y uso de Nuevas Tecnologías de la Información y Comunicación en profesores de cinco establecimientos chilenos de educación básica y media. Universitas Psychologica,11(2), 511521. Recuperado de http://www.javeriana.edu.co/universitaspsychologica/articulo.php? art=302

Cabero, J. (Dir.) (2014). La formación del profesorado en TIC: Modelo TPACK. Sevilla: Secretariado de Recursos audiovisuales y Nuevas Tecnologías de la Universidad de Sevilla.

Cabero, J. y Barroso, J. (2016). ICT teacher training: a view of the TPACK model / Formación del profesorado en TIC: una visión del modelo TPACK. Cultura y Educación, 28(3), 633-663. Recuperado de http://dx.doi.org/1 $0.1080 / 11356405.2016 .1203526$

Cabero, J., Marín, V. y Castaño, C. (2015). Validación de la aplicación del modelo TPACK para la formación del profesorado en TIC. @tic. Revista d'Innovació Educativa, 14, 13-22. https://doi.org/10.7203/attic.14.4001

Cengiz, C. (2014). The development of TPACK, Technology Integrated Self-Efficacy and Instructional Technology Outcome Expectations of pre-service physical education teachers. Asia-Pacific Journal of Teacher Education, 43 (5), 411-422. https://doi.org/10.1080/1359866X.2014.932332

Cózar, R., Zagalaz, J. y Sáez, J. M. (2015). Creating digital curricular contents of Social Sciences for Primary Education. A TPACK experience for future teachers. Educatio Siglo XXI, 33(3), 147-167.http://dx.doi. org $/ 10.6018 / \mathrm{j} / 240921$

Durán-García, M. E. y Durán-Aponte, E. E. (2013). Conceptos de calor y trabajo en un foro electrónico. Efectos de la autoeficacia computacional. Educación Química, 24(2), 247-254. Recuperado de http://www.sciencedirect.com/science/article/pii/S0187893X13724698

Fernández-Díaz, E. y Calvo, A. (2012). In-service teacher education in the innovatory use of ICT. An action research in Pre-school and Primary Education. Profesorado. Revista de Currículum y Formación del Profesorado, 16(2), 403-418. Recuperado de http://recyt.fecyt.es/index.php/profesorado/issue/view/2345

Howard, M. C. (2014). Creation of a Computer Self-Efficacy Measure: Analysis of internal consistency, psychometric properties, and validity. Cyberpsychology, Behavior, and Social Networking, 17(10), 677-681. https:// doi.org/10.1089/cyber.2014.0255

Jiménez-Cortés, R., Rebollo-Catalán, A., García-Pérez, R. y Buzón-García, O. (2015). Motivos de uso de las redes sociales virtuales: Análisis de perfiles de mujeres rurales. RELIEVE, 21(1), 1-17. https://doi.org/10.7203/ relieve.21.1.5153 
Juniu, S. (2011). Pedagogical uses of technology in physical education. Journal Education, Recreation y Dance, 82(9), 41-49. https://doi.org/10.1080/07303084.2011.10598692

Ladrón-de-Guevara, L., Almagro, B. J. y Cabero, J. (en revisión). Conocimiento tecnológico, pedagógico y disciplinar (TPACK) en docentes de Educación Física. Manuscrito en proceso de revisión para su publicación.

López, O., Sanabria, L. B. y Sanabria, M. (2014). Logro de aprendizaje en ambientes computacionales: autoeficacia, metas y estilo cognitivo. Psicología desde el Caribe, 31(3), 475-494. https://doi: 10.14482/psdc.31.3.5366

Mishra, P. y Koehler, M. J. (2006). Technological pedagogical content knowledge: A framework for teacher knowledge. Teachers College Record, 108(6), 1017-1054. https://doi: 10.1111/j.14679620.2006.00684.x

Mishra, P. y Koehler, M. J. (2008). Introducing technological pedagogical content knowledge. Paper presented at the Annual Meeting of the American Educational Research Association, New York, March 1-16. (Conference Presentation).

Pérez-Rodríguez, M. A., Aguaded, J. I. y Fandos, M. (2010). A properly policy and the permanent teacher's training, key in the ITC Centre impulse in Andalusia (Spain). Estudios Pedagógicos, 35(2), 137-154. https://doi: 10.4067/S0718-07052009000200008

Pla-Campàs, G. y Juncà, A. (2015). Hacia la digitalización ideal del profesorado de educación física. Tándem. Didáctica de la Educación Física, 49.

Prat, Q., Camerino, O. y Coiduras, J. LL. (2013). Introducción de las TIC en educación física. Estudio descriptivo sobre la situación actual. Apunts. Educación Física y Deportes, 113(3), 37-44. https://doi: 10.5672/ apunts.2014-0983.es.(2013/3).113.03

Rebollo-Catalán, A. y Vico-Bosch, A. (2014). El apoyo social percibido como factor de inclusión digital de las mujeres de entorno rural en las redes sociales virtuales. Comunicar, 43(22), 173-180. https://doi: 10.3916/C432014-17

Rebollo-Catalán, A., Vico-Bosch, A. y García-Pérez, R. (2015). El aprendizaje de las mujeres de las redes sociales y su incidencia en la competencia digital. Revista Prisma Social, 15, 122-146. Recuperado de http://www. isdfundacion.org/publicaciones/revista/numeros/15/secciones/tematica/t_04_mujeres-rrss.html

Roig, R. y Flores, R. (2014). Conocimiento tecnológico, pedagógico y disciplinario del profesorado: el caso de un centro educativo inteligente. EDUTEC: Revista Electrónica de Tecnología Educativa, 47, 1-17. http://dx.doi. org/10.21556/edutec.2014.47.93

Schmidt, D., Baran, E., Thompson, A., Mishra, P., Koehler, M. J. y Shin, T. (2009). Technological pedagogical content knowledge (TPACK): The development and validation of an assessment instrument for preservice teachers. Journal of Research on Technology in Education, 42(2), 123-149..

Semiz, K. y Ince, M. L. (2012). Pre-service physical education teachers' technological pedagogical content knowledge, technology integration self-efficacy and instructional technology outcome expectations. Australasian Journal of Educational Technology, 28(7), 1248-1265.

Sevillano, M. L. y Fuero, R. (2013). Initial ICT training for teachers: An analysis in Castilla-La Mancha. Profesorado, 17(3), 151-183. Recuperado de http://recyt.fecyt.es/index.php/profesorado/issue/view/2342

Shulman, L. S. (1987). Knowledge and teaching: Foundations of the new reform. Harvard Educational Review, 57 (1), 1-22. 


\section{NOTA}

1. Usamos la denominación profesor de Educación Primaria en lugar de maestro de Educación Primaria dado que los grados universitarios en España titulan por igual la formación inicial de todo tipo de docentes dentro de la educación reglada.

\section{INFORMACIÓN SOBRE LOS AUTORES}

Carlos Fernández-Espínola. Graduado en Educación Primaria con mención en Educación Física (CEU Cardenal Spínola) y Máster en Educación Físico-Deportiva (Universidad de Huelva). Doctorando de la Universidad de Huelva y está contratado como personal investigador no doctor en la Universidad de Huelva. Su principal línea de investigación está vinculada con la enseñanza de la Educación Física y las nuevas tecnologías.

$\triangle$ carlos.fernandez@ddi.uhu.es

Laura Ladrón de Guevara. Diplomada en Magisterio Educación Física (CEU Cardenal Spínola, licenciada en Ciencias de la Actividad Física y el Deporte (Universidad de Granada) y Máster Oficial en Educación y TIC ELearning (Universidad Abierta de Cataluña). Doctoranda de la Universidad de Sevilla y Profesora del Departamento de Actividad Física y Deporte del CEU Cardenal Spínola CEU (adscrito a la Universidad de Sevilla). Su principal línea de investigación está relacionada con las nuevas tecnologías y la Educación Física.

\section{lguevara@ceuandalucia.es}

Bartolomé Jesús Almagro. Licenciado en Ciencias de la Actividad Física y del Deporte (Universidad de Granada) y Doctor por la Universidad de Huelva con Mención Europea. Profesor Ayudante Doctor de la Universidad de Huelva. Director del Máster Universitario en Investigación en Educación Física y Ciencias del Deporte. Sus principales líneas de investigación versan sobre enseñanza, motivación, nuevas tecnologías y validación de instrumentos de medida para el ámbito de la actividad física y el deporte.

\section{almagro@dempc.uhu.es}

José Antonio Rebollo. Doctor por la Universidad de Huelva. Profesor Asociado de la Universidad de Huelva (Departamento de Didácticas Integradas) y Funcionario de la Consejería de Educación de la Junta de Andalucía. Su actual actividad docente universitaria se centra en la Enseñanza de la Educación Física en el Grado de Educación Infantil. Sus principales líneas de investigación giran en torno a la Educación Física y el Deporte. Es autor de publicaciones en revistas y libros, y ha dirigido tesis doctorales relacionadas con el área.

\section{joseantonio.rebollo@dempc.uhu.es}

